\title{
ON THE ORBITS OF G-CLOSURE POINTS OF ULTIMATELY NONEXPANSIVE MAPPINGS
}

\author{
MO TAK KIANG
}

Received 24 March 2006; Accepted 12 April 2006

Let $X$ be a closed subset of a Banach space and $G$ an ultimately nonexpansive commutative semigroup of continuous selfmappings. If the $G$-closure of $X$ is nonempty, then the closure of the orbit of any $G$-closure point is a commutative topological group.

Copyright (c) 2006 Mo Tak Kiang. This is an open access article distributed under the Creative Commons Attribution License, which permits unrestricted use, distribution, and reproduction in any medium, provided the original work is properly cited.

\section{Introduction}

Let $(X, d)$ be a metric space. A mapping $f: X \rightarrow X$ is called nonexpansive if for every $x, y \in X$, we have $d(f(x), f(y)) \leq d(x, y)$. Edelstein introduced in [2] the concept of $f$ closure points for nonexpansive mappings and proved that a nonexpansive mapping of $\mathbb{E}^{n}$ admits a fixed point if it has a nonempty set of $f$-closure points (points which are cluster points of $\left\{f^{n}(x)\right\}$ for some $\left.x \in X\right)$.

When $G$ is a family of mappings $g: X \rightarrow X$ forming a semigroup under composition, the notion of $G$-closure points of $X$ was introduced in [5] to generalize the concept of $f$-closure point. A $G$-closure point $x$ of $X$ is a cluster point of an orbit $G(z)$ for some $z \in X$. The study of $f$-closure points sets (called $\omega$-limit sets in $[1,7]$ ), orbits, and $G$ closure points (e.g., $[3,4,6])$ has since been of great interest in the fixed points theorems for various contractive-type mappings. In [7], Roehrig and Sine showed that when $C$ is a closed set in a Banach space $B$ and $f: C \rightarrow C$ a nonexpansive mapping, suppose for some $x \in C$, the $\omega$-limit set $S$ (i.e., the set of $f$-closure points) of $x$ is nonempty, then there exists a binary operation in the set $S$ under which it is a monothetic topological group in the topology induced by the metric of $B$. It is the purpose of this paper to show that when $G$ is a commutative ultimately nonexpansive semigroup of mappings (a concept introduced by Edelstein and the author in $[3,4])$ of a closed subset $X$ of a Banach space into itself and if there is a $G$-closure point $z \in X$, then there exists a binary operation in the closure of the orbit of $z$ such that it is a commutative topological group. 
2 G-closure points of ultimately nonexpansive mappings

\section{Definitions and notations}

Definition 2.1. Let $(X, d)$ be a metric space and $G: X \rightarrow X$ a semigroup of mappings. For any $x \in X$, the set $G(x)=\{g(x): g \in G\}$ is called the orbit of $x$ under $G$.

Definition 2.2. A semigroup of selfmappings $G$ of a metric space $(X, d)$ is called asymptotically nonexpansive if for all $x, y \in X$ there exists $g \in G$ such that for all $f \in G, d(f g(x)$, $f g(y)) \leq d(x, y)$.

Definition 2.3. A semigroup $G$ of continuous selfmappings of a metric space $(X, d)$ is called ultimately nonexpansive if for every pair of points $x, y \in X$ and for every $\alpha>0$ there is $g \in G$ such that for all $f \in G, d(f g(x), f g(y)) \leq(1+\alpha) d(x, y)$. (When $\alpha=0, G$ is asymptotically nonexpansive.)

Definition 2.4. Let $f:(X, d) \rightarrow(X, d)$. Then the $\omega$-limit set of $x$ (denoted by $\omega(x)$ in $[1,7])$ or the $f$-closure of $x$ (denoted by $X^{f}$ in [2]) is the set

$$
\left\{y \in X: y=\lim _{n \in N_{1}} f^{n}(x)\right\}
$$

where $N_{1}$ is a strictly increasing sequence in $\mathbb{Z}^{+}$.

Definition 2.5. Let $G$ be a family of mappings of $(X, d)$ into itself. The $G$-closure of $X$ consists of all points $x \in X$ such that for some $z \in X$, any $\varepsilon>0$, and any $f \in G$, there is a $g \in G$ such that $d(f g(z), x)<\varepsilon$. The $G$-closure of $X$ is denoted by $X^{G}$.

Definition 2.6. A point $x$ of $(X, d)$ is called G-recurrent (or recurrent under $G$ ) if for any $\varepsilon>0$ and any $f \in G$, there is a $g \in G$ such that $d(f g(x), x)<\varepsilon$.

\section{Preliminaries}

In the following, $G$ is a family of ultimately nonexpansive commutative semigroups of continuous mappings of a metric space $(X, d)$ into itself.

Proposition 3.1. If $X^{G} \neq \varnothing$ and $z \in X^{G}$, then for all $f \in G$, for all $\varepsilon>0$, there exists $g \in G$ with $d(f g(z), z)<\varepsilon$.

Proof. See [3, Proposition 1(a)].

Proposition 3.2. If $z \in X^{G}$, then $\left.G\right|_{G(z)}$ is a family of asymptotically nonexpansive mappings.

Proof. See [3, Proposition 2(a)].

Proposition 3.3. If $z \in X^{G}$, then $\left.G\right|_{G(z)}$ is a family of isometries.

Proof. By Proposition 3.2, $\left.G\right|_{G(z)}$ is a family of asymptotically nonexpansive mappings. By a result of Holmes and Narayanaswami (see [5, Proposition 2]), $\left.G\right|_{G(z)}$ is a family of isometries. 
Corollary 3.4. If $z \in X^{G}$, then $\left.G\right|_{\overline{G(z)}}$ is a family of isometries.

Proof. Obvious.

Proposition 3.5. When $(X, d)$ is complete and $z \in X^{G}$, then for each $f \in G, f(\overline{G(z)})=$ $\overline{G(z)}$. That is, each $f$ is an onto mapping when restricted to $\overline{G(z)}$.

Proof. For each $f \in G$, clearly $f \overline{G(z)} \subseteq \overline{f G(z)} \subseteq \overline{G(z)}$ since $f$ is continuous. It suffices to show that $\overline{G(z)} \subseteq f \overline{G(z)}$. Let $p \in \overline{G(z)}$. Then for all $\varepsilon=1 / n$, there exists $g_{n} \in G$ such that $d\left(g_{n}(z), p\right)<1 / 2 n$.

Since $z \in X^{G}$, for the above $f$ and $g_{n}$, there exists $t_{n}$ corresponding to $f g_{n}$ such that $d\left(f g_{n} t_{n}(z), z\right)<1 / 2 n$. By Proposition 3.3, each member in $G$ is an isometry on $G(z)$. Hence $d\left(f g_{n} g_{n} t_{n}(z), p\right) \leq d\left(f g_{n} g_{n} t_{n}(z), g_{n}(z)\right)+d\left(g_{n}(z), p\right)<1 / 2 n+1 / 2 n=1 / n$. Let $h_{n}=$ $g_{n} g_{n} t_{n}$. Then for each $\varepsilon=1 / n$, there exists $h_{n} \in G$ such that $d\left(f h_{n}(z), p\right)<1 / n$. Now $\left\{f h_{n}(z)\right\}$ converges to $p$ implies that $\left\{h_{n}(z)\right\}$ is a Cauchy sequence since $f$ is an isometry. Since $X$ is complete $\left\{h_{n}(z)\right\}$, converges to a point $q \in \overline{G(z)}$.

Clearly $f(q)=f\left(\lim _{n \rightarrow \infty} h_{n}(z)\right)=\lim _{n \rightarrow \infty} f h_{n}(z)=p$, showing that $\overline{G(z)} \subseteq f \overline{G(z)}$.

Proposition 3.6. For each $f \in G,\left.f\right|_{\overline{G(z)}}$ is a homeomorphism.

Proof. By the corollary to Propositions 3.3 and 3.5, each $f$ is an isometry of $\overline{G(z)}$ onto itself. Hence, each $f$ is a homeomorphism.

\section{Main result}

Theorem 4.1. Let $X$ be a closed subset of a Banach space and let $G: X \rightarrow X$ be a commutative semigroup (under composition) of ultimately nonexpansive mappings. If $X^{G} \neq \varnothing$ and $z$ is any arbitrary member in $X^{G}$, then a binary operation can be introduced in $\overline{G(z)}$ such that $\overline{G(z)}$ is a commutative topological group in the topology induced by the metric of $X$.

Proof. By Proposition 3.6, each $f \in G$ is an isometry and therefore a homeomorphism of $\overline{G(z)}$ onto itself. Hence, the inverse of each $f \in G$ exists. Let $f^{-1}$ denote the inverse of $f$. By Proposition 3.1, since $z \in X^{G}$, for each $\varepsilon=1 / n$, for the above $f$, there exists $f_{n} \in G$ such that $d\left(f f_{n}(z), z\right)<1 / n$. Denote $g_{n}=f f_{n}$. We have $\lim _{n \rightarrow \infty} g_{n}(z)=z$. Let $p, q \in \overline{G(z)}$. Then there exist $h_{n} \in G$ and $t_{n} \in G$ such that $\lim _{n \rightarrow \infty} h_{n}(z)=p$ and $\lim _{n \rightarrow \infty} t_{n}(z)=q$. Denote $h_{n}^{*}=h_{n} g_{n}^{-1}$ and $t_{n}^{*}=t_{n} g_{n}^{-1}$. Then $h_{n}=h_{n}^{*} g_{n}$ and $t_{n}=t_{n}^{*} g_{n}$.

Define $q \circ p=\lim _{n \rightarrow \infty} t_{n}^{*} g_{n} h_{n}^{*}(z)$. This limit exists since each member of $G$ is an isometry. It is also unique. Clearly $q \circ p \in \overline{G(z)}$. The following results are immediate:

(1) the operation $\circ$ is associative,

(2) $z$ is the identity of $\overline{G(z)}$ ( since $z \circ p=\lim _{n \rightarrow \infty} g_{n}^{*} g_{n} h_{n}^{*}(z)=\lim _{n \rightarrow \infty} h_{n}(z)=p$ ),

(3) $q \circ p=p \circ q$ since $G$ is commutative.

If $p=\lim _{n \rightarrow \infty} h_{n}(z)=\lim _{n \rightarrow \infty} h_{n}^{*} g_{n}(z)$, define $p^{-1}=\lim _{n \rightarrow \infty} g_{n}\left(h_{n}^{*}\right)^{-1}(z)$. This limit exists as each member of $G$ is an isometry; clearly $p^{-1} \circ p=\lim _{n \rightarrow \infty}\left(h_{n}^{*}\right)^{-1} g_{n} h_{n}^{*}(z)=z=$ $p \circ p^{-1}$. Hence $\overline{G(z)}$ is a commutative group.

Next, let $p_{i} \rightarrow p$ and $q_{i} \rightarrow q$, where $p_{i}, q_{i}, p, q \in \overline{G(z)}$. Then there exist $h_{i, n}$ and $t_{i, n}$ such that $\lim _{n \rightarrow \infty} h_{i, n}(z)=p_{i}$ and $\lim _{n \rightarrow \infty} t_{i, n}(z)=q_{i}$. Denote $h_{i, n}^{*}=h_{i, n} g_{n}^{-1}$ and $t_{i, n}^{*}=t_{i, n} g_{n}^{-1}$. 


\section{G-closure points of ultimately nonexpansive mappings}

Then $\left(t_{i, n}^{*}\right)^{-1}=g_{n} t_{i, n}^{-1}$. Since $\left(t_{n}^{*}\right)^{-1}=g_{n} t_{n}^{-1}, q^{-1}=\lim _{n \rightarrow \infty} g_{n}\left(t_{n}^{*}\right)^{-1}(z)$, and $q_{i}^{-1}=$ $\lim _{n \rightarrow \infty} g_{n}\left(t_{i, n}^{*}\right)^{-1}(z)$, we have

$$
\begin{aligned}
\left\|p_{i} \circ q_{i}^{-1}-p \circ q^{-1}\right\| \leq & \left\|q_{i}^{-1} \circ p_{i}-q^{-1} \circ p_{i}\right\|+\left\|p_{i} \circ q^{-1}-p \circ q^{-1}\right\| \\
= & \left\|\lim _{n \rightarrow \infty}\left(t_{i, n}^{*}\right)^{-1} g_{n} h_{i, n}^{*}(z)-\lim _{n \rightarrow \infty}\left(t_{n}^{*}\right)^{-1} g_{n} h_{i, n}^{*}(z)\right\| \\
& +\left\|\lim _{n \rightarrow \infty} h_{i, n}^{*} g_{n}\left(t_{n}^{*}\right)^{-1}(z)-\lim _{n \rightarrow \infty} h_{n}^{*} g_{n}\left(t_{n}^{*}\right)^{-1}(z)\right\| \\
= & \left\|\lim _{n \rightarrow \infty} g_{n}\left(t_{i, n}^{*}\right)^{-1}(z)-\lim _{n \rightarrow \infty} g_{n}\left(t_{n}^{*}\right)^{-1}(z)\right\| \\
& +\left\|\lim _{n \rightarrow \infty} h_{i, n}^{*}(z)-\lim _{n \rightarrow \infty} h_{n}^{*}(z)\right\| \\
= & \left\|\lim _{n \rightarrow \infty} g_{n}\left(t_{i, n}^{*}\right)^{-1}(z)-\lim _{n \rightarrow \infty} g_{n}\left(t_{n}^{*}\right)^{-1}(z)\right\| \\
& +\left\|\lim _{n \rightarrow \infty} h_{i, n} g_{n}^{-1}(z)-\lim _{n \rightarrow \infty} h_{n} g_{n}^{-1}(z)\right\| \\
= & \left\|q_{i}^{-1}-q^{-1}\right\|+\left\|\lim _{n \rightarrow \infty} h_{i, n}(z)-\lim _{n \rightarrow \infty} h_{n}(z)\right\| \\
= & \left\|q_{i}^{-1}-q^{-1}\right\|+\left\|p_{i}-p\right\|,
\end{aligned}
$$

since all mappings are isometries.

As $i \rightarrow \infty,\left\|q_{i}^{-1}-q^{-1}\right\|$ and $\left\|p_{i}-p\right\|$ become arbitrarily small, so $\left\|p_{i} \circ q_{i}^{-1}-p \circ q^{-1}\right\|$ approaches zero. Hence the operation $\circ$ is continuous in both variables and $\overline{G(z)}$ is a topological group.

\section{References}

[1] C. M. Dafermos and M. Slemrod, Asymptotic behavior of nonlinear contraction semigroups, Journal of Functional Analysis 13 (1973), no. 1, 97-106.

[2] M. Edelstein, On nonexpansive mappings, Proceedings of the American Mathematical Society 15 (1964), no. 5, 689-695.

[3] M. Edelstein and M. T. Kiang, On ultimately nonexpansive semigroups, Pacific Journal of Mathematics 101 (1982), no. 1, 93-102.

[4] __ A common fixed-point theorem in reflexive locally uniformly convex Banach spaces, Proceedings of the American Mathematical Society 94 (1985), no. 3, 411-415.

[5] R. D. Holmes and P. P. Narayanaswami, On asymptotically nonexpansive semigroups of mappings, Canadian Mathematical Bulletin 13 (1970), 209-214.

[6] M. A. Khamsi, M. Lin, and R. Sine, On the fixed points of commuting nonexpansive maps in hyperconvex spaces, Journal of Mathematical Analysis and Applications 168 (1992), no. 2, 372380.

[7] S. F. Roehrig and R. C. Sine, The structure of $\omega$-limit sets of nonexpansive maps, Proceedings of the American Mathematical Society 81 (1981), no. 3, 398-400.

Mo Tak Kiang: Department of Mathematics and Computing Science, Saint Mary's University, Halifax, Nova Scotia, Canada B3H 3C3

E-mail address: motak.kiang@smu.ca 